\title{
Lessons Learned From a Failed Implementation: Effective Communication with Patients in Transmission-Based Precautions
}

\section{Luize Fábrega Juskevicius ( $\square$ luizejuskevicius@usp.br)}

Sao Paulo University College of Nursing: Universidade de Sao Paulo Escola de Enfermagem https://orcid.org/0000-0002-5414-745X

\section{Reginaldo Adalberto Luz}

Faculdade de Ciências Médicas da Santa Casa de São Paulo: Faculdade de Ciencias Medicas da Santa Casa de Sao Paulo

\section{Adriana Maria da Silva Felix}

Faculdade de Ciências Médicas da Santa Casa de São Paulo: Faculdade de Ciencias Medicas da Santa Casa de Sao Paulo

\section{Stephen Timmons}

University of Nottingham

\section{Maria Clara Padoveze}

USP EE: Universidade de Sao Paulo Escola de Enfermagem

\section{Research}

Keywords: Health Communication, Implementation Science, Transmission-Based Precautions

Posted Date: February 14th, 2022

DOI: https://doi.org/10.21203/rs.3.rs-1280711/v1

License: (c) (1) This work is licensed under a Creative Commons Attribution 4.0 International License. Read Full License 


\section{Abstract \\ Background}

Patient education and engagement about standard and transmission-based precautions can be one important strategy to prevent adverse events related to isolation. However, most patient education is still highly prescriptive, and is thus unlikely to help patients to engage in their own care. Implementing effective communication between health workers and patients requires behavior change, leading to a meaningful dialogue between the parties involved in the education process.

\section{Objective}

this study aimed to evaluate the process of implementation of a protocol for effective communication with patients in transmission-based precautions (Com-Efe).

\section{Methods}

This is implementation researchusing qualitative methods, carried out in four sequential phases: 1) nonparticipant observation in inpatient wards, with the objective of assessing the context; 2) designing the intervention for the implementation of the protocol Com-Efe, based on the results obtained in the previous phase; 3 ) preliminary assessment and adaptation of the Com-Efe protocol, through workshops with nurses; 4) final assessment of the implementation results, through semi-structured interviews with nurses. The study was performed in a public, secondary level, teaching hospital. The Consolidated Framework for Implementation Research (CFIR) was used as the reference for both interview design and data analysis, aiming to identify barriers and enablers of the implementation process.

\section{Results}

The main factors that could have facilitated adherence were beliefs and perceived advantages in using the Com-Efe. The main barriers which may have contributed to the failure of implementation were the unfavorable climate for implementation, insufficient individual and leadership commitment, and lack of understanding of the concepts underpinning effective communication. The participants recognized they had low adherence to the Com-Efe protocol.

\section{Conclusions}

Despite using a systematic approach, the Com-Efe protocol was not fully implemented. The lessons learned in this study allowed us to propose suggestions for future implementations in similar contexts. 
Among these suggestions are to develop strategies to generate awareness of patient-centered care, maintaining patient autonomy, and seeking dialog for patient engagement.

\section{Background}

Standard precautions (SP) and transmission-based precautions (TBP) are fundamental for the prevention and control of the spread of microorganisms ${ }^{1}$. Although the benefits of TBP to prevent the spread of microorganisms are recognized, individuals in TBP are exposed to risks related to isolation measures. Results of a systematic review showed evidence of negative effects on the psychological well-being of patients, such as changes in mood, fatigue, anxiety, depression, among others ${ }^{2}$. Other clinical studies also show that individuals in TBP have greater dissatisfaction with their care ${ }^{3}$, greater risk of medicationrelated errors ${ }^{4}$, and longer hospital stays when compared to patients who were not isolated ${ }^{5}$.

In recent years, the development of institutional policies aimed at health education, through effective communication, have become a key part of promoting the engagement of patients in their care ${ }^{6-13}$. To support this educational action, in a previous study we developed and validated a protocol to promote effective communication with patients inTBP. This protocol, called "Com-Efe", aims to provide professionals with the tools for the development of educational actions for individuals in TBP, in order to reducing their vulnerability to adverse events related to TBP. The Com-Efe is not intended to be used merely as an adjuvant to the traditional health education process, but rather to support a change of approach to a more dialogic action, considering the individual's autonomy and respecting their prior knowledge ${ }^{7}$.

This proposed change in approach can be considered a complex intervention in health services.

Therefore, we sought to use the tools of implementation science, by identifying barriers and facilitators to design the strategy for implementing the Com-Efe protocol in a university hospital. This study aimed to develop and evaluate the process and results of implementing the protocol for effective communication with hospitalized patients in a TBP situation (Com-Efe).

\section{Methods}

\section{Study design}

This is a study on the implementation of a protocol for effective communication with hospitalized patients inTBP (Com-Efe) ${ }^{7}$, using multiple qualitative methods. The theoretical framework to describe and analyze the implementation process was the Consolidated Framework for Implementation Research CFIR ${ }^{14}$. The study was developed in four phases (Figure 1. See Additional file) in order to answer the research questions: "How does the process of implementing Com-Efe in hospital happen?", "What are the barriers and facilitators for implementing Com-Efe?".

\section{Setting}


The study was carried out in the medical and surgical care wards of a teaching hospital located in the city of São Paulo, Brazil, with approximately 200 beds.

\section{Participants}

Nurses who worked in medical and surgical care wards and in the Hospital Infection Control Service.

\section{Implementation phases and data collection}

The implementation process was carried out in sequential phases, as described below:

Phase 1: Context familiarization - the familiarization of the context was conducted in April and May 2018. In order to deepen the understanding of the context in which patient education for TBP was carried out, non-participant observation was chosen. Information about the context was collected over 20 nonsequential hours ( 10 observation sessions with an average of 2 hours each) and recorded in a field diary by one of the researchers (L.F.J.). The researcher placed herself in several strategic observation locations, such as the prescription area, the medication room, the procedure room, hallways, bedrooms, living rooms, administrative rooms, and the dining room.

Phase 2: Intervention - Initially, Com-Efe was adapted to the official format for operational protocols of the study institution and later inserted in the online training system. Additionally, an expository class on the subject was offered to nurses working at the site. The materials were available for 18 days. After this period, the researcher (L.F.J.) conducted on-site training for all shifts of the units involved, in order to clarify questions about the materials available on the online platform, and to raise awareness among the professionals involved. The training was carried out with a focus on the concepts of health education and vulnerability - concepts used to design Com-Efe. After the face-to-face training, the following support materials were made available at the units: Com-Efe protocol printed in the hospital's standard format, Com-Efe advertising banner, and an effective communication stamp to be affixed to the patient's medical record after the approach has been completed using Com-Efe.

Phase 3: Preliminary assessment and adaptation - to analyze the implementation and identify the necessary adaptations, workshops were held with nurses, led by one of the researchers (L.F.J.), using a question scriptin order to identify barriers and facilitators in the implementation process of the Com-Efe. The choice of questions was based on the relevance and importance of CFIR constructs for this stage of Com-Efe adaptation. Therefore, the following constructs were used: intervention origin, complexity, relative advantage, and compatibility. The workshops lasted 20 minutes each; the participants received and signed an informed consent form. The workshop results were recorded and transcribed verbatim.

Phase 4: Final assessment - the final assessment of the implementation process was carried out through semi-structured interviews, carried out by telephone with nurses from the units involved. The interviews were recorded and transcribed verbatim.

The interviews were carried out by one of the researchers (L.F.J.), after the interviewees had signed a consent form, and then recorded and transcribed verbatim. 


\section{Data analysis}

Data analysis was performed using descriptive analysis (phase 1) and thematic content analysis of the qualitative data (phases 2 and 4). The data collected in phase 1 contained in the field diary were initially organized in the form of a hand-written text to facilitate the discussion among researchers. We identified relevant aspects representative of the relationships and interactions between health professionals and other individuals in the context. These selected aspects were classified according to the CFIR, focusing on the domains "characteristics of individuals" and "internal setting" 14-16. For the analysis of data from the workshops and semi-structured interviews, thematic content analysis was used as described by Bardin $(2016){ }^{17}$. After the reading of the transcript, the coding was performed considering the CFIR domains as the categories and attributing the registration units found to their respective constructs, according to the inclusion and exclusion criteria for the analysis of CFIR constructs ${ }^{14}$. In the last stage of data processing, the inference and interpretation of the collected and categorized information was performed, exploring the meanings attributed to the categories.

The study followed the steps recommended in the Consolidated Criteria for Reporting Qualitative Research (COREQ) ${ }^{18}$.

\section{Results}

\section{Phase 1: Context familiarization}

At this stage, it was sought to understand the relationships and interactions between the HCW and other individuals in the healthcare environment, mainly to identify potential barriers and facilitators for the implementation of Com-Efe. Ten observation sessions were performed, with an average of two hours for each moment in April and May 2018.

The total number of hospital beds available at the time of data collection was 34 and 31 in the Surgical and Medical Clinics respectively. At the time of observation, there were 10 patients in TBP in the wards, with 7 nursing professionals at the Medical Clinic and 9 nursing professionals at the Surgical Clinic.

In the Surgical Clinic ward, no atypical activities or situations that could interfere with the work process were witnessed. In the Medical Clinic ward, there was great movement during all the observed moments, with activities performed by different categories of HCW. It was observed that the nurse played a leadership role in the context and this could be demonstrated through the intensity of interaction with the other HCW in the context. The nurse represented a reference for all who are present in the context, but as the patient cannot move outside their room, the interaction with them is less intense than the relationship between the nurse and the visitors/companions.

Through the observations notes in the field diary, elements emerged that were classified as facilitators or barriers, and distributed to the domains and constructs of the CFIR (Table 1. See Additional file 1). We 
identified four main stakeholder groups (nursing supervisors, infection prevent and control team, nurses and patients). It was observed that all these identified groups had potential high impact in influencing the context, while the intervention had great significance in their routine/health, once the Com-Efe protocol is implemented. 
Table 1

Classification of barriers and facilitators identified during the implementation effective communication (Com-Efe). São Paulo, Brazil, 2021.

\section{INTERVENTION CHARACTERISTICS (CFIR Domain)}

\section{CFIR Construct Barriers Enablers Quotations (examples)}

\begin{tabular}{|c|c|c|}
\hline $\begin{array}{l}\text { Intervention } \\
\text { Source }\end{array}$ & $x$ & $\begin{array}{l}\text { "I believe that it is based on evidence; just the fact that it } \\
\text { comes from a researcher at the School of Nursing, with } \\
\text { all the requirements there, is already based on this } \\
\text { principle" }-12 P 4\end{array}$ \\
\hline
\end{tabular}

Evidence $\quad \mathrm{N} \quad$ "Well, I think this implementation comes to add a better Strength and $\quad$ quality of care, especially for patients in TBP, mainly at
Quality
the time of the pandemic, where we have these TBP involved, then you bring quality not only to the professional, but to the patient and family." - I3P4

Relative
Advantage

"By the methodology of the Com-Efe protocol, I Advantage understand that it is a more systematized logic, with the steps you must follow, what are the steps, compared to what we did before Com-Efe; of course we gave the orientation but it happened in a not so standardized way." - I3P4
Adaptability
$\mathrm{X}$
"I think it has to be put on computers and on TV reminders, because here at the hospital we have people who work with video, this could also be put in hospitalization area" - I1P4

Complexity $\quad \mathrm{X}$

"[...] what we felt was that during our work, because of the routine, we were in a hurry to do everything, and not using it as it should." - I2P4

Design Quality $\quad X$

"And what I think about the implementation of this stamp and Packaging (effective communication stamp in TBP) really is that it is not very useful" - I3P3

\section{OUTER SETTING (CFIR Domain)}

\section{CFIR Construct Barriers Enablers Quotations (examples)}

Cosmopolitanism $\quad \quad$ As far as I know, no other place was contacted to talk
about Com-Efe"-I2P4

Patient's Needs $\quad \mathrm{X}$ and Resources
[...] "one of the nurses' complaints is the lack of control by the family, and maybe it's lack of guidance. It is difficult for a lay person to understand that a bacterium they do not see can be harmful to other patients. And this is of little importance, because they are only concerned about their family members" - I5P4

1 CFIR: Consolidated Framework for Implementation Research. I: Interviewee. P: Phase.

Source: author. 


\section{INTERVENTION CHARACTERISTICS (CFIR Domain)}

Peer Pressure $\quad$ X "I think when you bring experiences with positive results from other places and present them before implementing, it makes a difference." - I3P4

\section{INNER SETTING (CFIR Domain)}

\section{CFIR Construct Barriers Enablers Quotations (examples)}

Structural $\quad \mathrm{X}$

Characteristics

$\mathrm{X}$
"Frequently there are individuals in TBP, mainly due to colonization/infection by multidrug-resistant microorganisms." - P1

"[...] there are many family members and companions, and then they stay mainly in the room with six patients, they all become friends, the family members, then they keep asking, want to help each other, so the first thing we do is to orient them about isolation, because that way, the family member who is in isolation, let's assume the companion who is already in a private room, I just put the sign, but this family member can no longer sit in the TV room, as he/she used to do, so the first thing is to tell this family member not to go to the TV room, not to go to the counter and keep putting their hands on the counter when talking to us." - I6P3

"Effective communication occurs in an insufficient way and suggests that the individual believes that everyone knows what should be done regarding HAl and TBP prevention measures."- $P 1$

Networks and $\quad \mathrm{X}$

Communications

Culture $\quad \mathrm{X} \quad \mathrm{X}$

Implementation $\quad \mathrm{X}$

Climate

$x$

"There is frequent training and continuous presence of students and researchers, which can be a favorable element for the permeability of professionals to innovations in care practices." - P1

"Lay people and doctors also do not use PPE correctly, what takes away our authority with the family" - I1P4

"[...] in the case of isolation, you go to the protocol, read, and along the way someone stops you, you have already forgotten the approach points. A simple thing to do, like going to the protocol, checking and seeing if I have oriented everything, can be very difficult." - I2P3

Tension for
Change

Relative Priority $\quad \mathrm{X}$
"I had no idea that it would be possible to do something systematized, we felt that maybe what we are doing was not the best." - I3P4

1 CFIR: Consolidated Framework for Implementation Research. I: Interviewee. P: Phase.

Source: author. 


\section{INTERVENTION CHARACTERISTICS (CFIR Domain)}

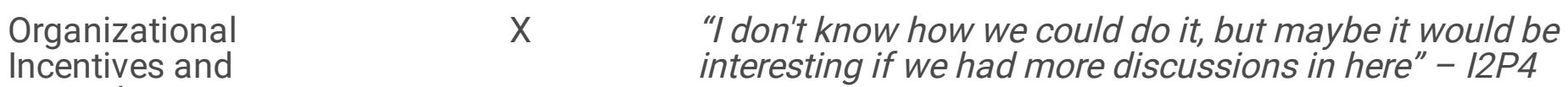

Rewards

Learning Climate $\quad$ "Work processes are possibly influenced by excessive
workload (due to lack of staff)." - P1

Leadership $\quad \mathrm{X}$

"At this moment we are not able to implement the

Engagement protocol, because in the training with you (researcher), we had to see where we were going to put the stamps (effective communication stamp in TBP) and I think that unfortunately that was lost; we said we were going to talk to the boss" - I5P3

$\begin{array}{ll}\text { Available } & X \quad \text { "I believe we have Moodle available, physical space for } \\ \text { Resources } & \text { meetings, employees' emails, and the sending of emails } \\ \text { to call for training." - I3P4 }\end{array}$

Access to $\quad X$

Knowledge and

"The work processes do not guarantee effective

Information communication between the health professionals of these services and patients, as they are not standardized (systematization of patient guidance)." - P1

\section{CHARACTERISTICS OF INDIVIDUALS (CFIR Domain)}

\section{CFIR Construct Barriers Enablers Qualifications}

Knowledge and $\quad \mathrm{X} \quad$ "Prevention measures exist and are widely adopted. It is Beliefs about the observed that there are still failures in full adoption, Intervention especially regarding hand hygiene and proper use of TBP, which can affect the way the patient is treated." - P1

Individual Stage $\quad X$

of Change

"I made little commitment, and I think that for myself and for the team; we could have been more dedicated to studying the material, especially now with the demand for Covid-19. To encourage colleagues in this critical period, we are seeing very different, very complex patients, so I think that in this situation, it would be difficult to try to change or encourage something. I think it would be possible, but in the current madness, it gets more complicated" - I2P4

Individual Identification "I consider myself a person very involved with the hospital activities, very committed to all the actions that with Organization

$X$ are proposed to me; I try to do my best." - I3P4

\section{PROCESS (CFIR Domain)}

\section{CFIR Construct Barriers Enablers Quotations (examples)}

1 CFIR: Consolidated Framework for Implementation Research. I: Interviewee. P: Phase.

Source: author. 


\section{INTERVENTION CHARACTERISTICS (CFIR Domain)}

\begin{tabular}{|c|c|c|}
\hline Planning & $X$ & $\begin{array}{l}\text { "I remember you bringing the protocol, explaining to us } \\
\text { how the steps would be and that we would have it printed } \\
\text { on the unit; at the beginning I remember that you brought } \\
\text { a little stamp to put on the medical records of patients } \\
\text { who we would approach, so I used it right away then; } \\
\text { later you came back to see the difficulties and then you } \\
\text { made the material more accessible; I don't remember if } \\
\text { there was any poster, and then there was a meeting with } \\
\text { the nurses and now this interview." - I2P4 }\end{array}$ \\
\hline Opinion Leaders & $x$ & $\begin{array}{l}\text { "[... I I think the HICC, the nurse or the doctors themselves - } \\
\text { that come to carry out surveillance and see if the patient } \\
\text { is in isolation, I think they are actors who can see if the } \\
\text { protocol is being followed, if the information is being } \\
\text { passed." - I4P3 }\end{array}$ \\
\hline Executing & $x$ & $\begin{array}{l}\text { "I think we haven't implemented it yet, I haven't done it in } \\
\text { any of the patients, nothing has changed, we're doing it } \\
\text { the same way."- I3P3 }\end{array}$ \\
\hline
\end{tabular}

1 CFIR: Consolidated Framework for Implementation Research. I: Interviewee. P: Phase.

Source: author.

\section{Phase 2 - Intervention}

After meetings with the stakeholders identified in the previous phase, the on-site training dissemination began. The invited participants were 14 nurses from the Medical Clinic and 14 nurses from the Surgical Clinic, including the nursing supervisors of the respective units, invited via email, with the Com-Efe attached with an indication for reading. However, the material was accessed for prior reading by only five nurses, representing $18 \%$ of the total participants.In total, 15 nurses from the medical and 9 from the surgical wards participated in the on-site training, corresponding to $100 \%$ of the nurses who were on duty at the training period. Nurses were encouraged to use the resources provided in the wards, and to apply the self-adhesive stamps to ensure the record of the Com-Efe approach in the patient' medical record.

The training was carried out in-person by a researcher with experience in teaching and TBP (L.F.J.), in all shifts for both wards. In total, 15 nurses from the Medical Clinic and 9 from the Surgical Clinic participated in the training, corresponding to $100 \%$ of the nurses who were on duty at the time the training was carried out.

After the in-person training, the following support materials were made available in the units: the printed Com-Efe protocol, a banner advertising the Com-Efe protocol, and Com-Efe adhesive stamps for affixing on medical recordswith the purpose of registering patients in TBP who received this approach.

\section{Phase 3: Preliminary assessment and adaptation}

The elements that emerged from the workshops were coded into 24 initial categories, organized into 17 intermediate categories, which remained as final thematic categories (TC), which in turn were distributed 
into the five domains of the CFIR. Barriers and facilitators were highlighted within each category inserted in the CFIR domains and constructs (Table 1).

After analyzing barriers and facilitators, the implementation process underwent adaptations related to the dissemination of its materials and concepts among the participants. Only the adaptable periphery of the Com-Efe was changed, without changing the protocol's core component, which is the concept of vulnerability. This considers the relationships with the patient in a dialectical process.

Adaptations were made to the training content and to the format of materials to be used with the patient. An educational video was developed, presenting the Com-Efe protocol and its advantages; the video was publishedon the institution's official website and also on social networks, in order to raise awareness among HCWs regarding Com-Efe and the essential concepts it is based on. In addition, a booklet was developed and delivered to the wards to support bedside guidance to patients in TBP.

\section{Phase 4: Final assessment}

The elements emerging in the final assessment interviews were coded into 8 initial categories, divided into 16 intermediate categories, which were organized into 25 final thematic categories, and later distributed in the domains and constructs of the CFIR. Barriers and facilitators were highlighted within each domain and construct of the CFIR (Table 1).

In the construct related to the patient's needs, the lack of dialogue between the HCW and the patient was identified as a barrier, which directly impacted the core element of Com-Efe. As facilitators, the perception of the need to guide patients and families about TBP for greater safety during hospitalization, in relation to adverse events related to TBP was identified. In the other constructs, the barriers identified most frequently were related to the adaptation of the material for institutional incorporation, such as fact that there was no formally appointed institutional leader to lead the implementation process, and to the lack of commitment of the leadership to the implementation of Com-Efe. Finally, an important barrier was the unfavorable climate for the implementation of prioritizing Com-Efe as an institutional interest.

It has to be noted that in some circumstances a given construct can be understood as a barrier or facilitator, depending on how the situation develops. In terms of the constructs of culture and structural characteristics, the existence of a safety culture from the patient in relation to Healthcare Associated Infection (HAl) and the existence of an adequate physical structure were pointed out.At the same time, there are still failures in adherence to TBP, among other failures related to HAl prevention.

Despite the use of qualitative multi-methods, some existing constructs in the CFIR could not be identified, such as: cost, testability, external incentive policies, readiness for implementation, self-efficacy, engagement, supporters, reflection and evaluation, and external agents of change.

The results obtained showed that there was no effective incorporation of Com-Efe as a routine tool to improve the process of engaging patients inTBP in their own care; thus, the implementation was not successful. The participants recognized they had low adherence to the Com-Efe protocol. Therefore, we 
identified the main lessons learned in this implementation process, and we propose suggestions to increase the chance of success in implementing Com-Efe in the future in similar contexts. The results are presented in a table according to the domain and construct of the CFIR (Table 2. See Additional file 2). 
Table 2

Lessons learned and suggestions for future strategies for implementing effective communication (ComEfe). São Paulo, Brazil, 2021.

\section{CFIR Domains Main lessons learned in the and Constructs implementation process \\ Suggestions for future implementation strategies}

\section{INTERVENTION CHARACTERISTICS}

Intervention

Source
The individuals did not develop a sense of belonging since they did not feel involved in the intervention proposal.

- Engage key stakeholders in TBP measures from the beginning of the implementation process.

- Identify opinion leaders to form partnerships for the implementation process from the beginning.

- Offer technical and scientific support throughout the implementation process.

\section{Evidence}

Strength and

Quality
Familiarization with robust scientific evidence has contributed to promoting the engagement of some key stakeholders.
- Develop innovative strategies, but as close as possible to what is already being done.

- Present robust scientific evidence and make the evidence available for consultation throughout the implementation process.

- Present external or internal examples of success using the same tool or similar tools.

- Present and discuss the benefits of the intervention to key stakeholders.

- Develop strategies to increase awareness of patient-centered care and preservation of patient's autonomy.

II. OUTER SETTING

1 CFIR: Consolidated Framework for Implementation Research. 


\section{CFIR Domains Main lessons learned in the and Constructs implementation process}

$\begin{array}{ll}\begin{array}{l}\text { Patient's Needs } \\ \text { and Resources }\end{array} & \begin{array}{l}\text { Professionals had different degrees } \\ \text { of perception about the health needs } \\ \text { of patients in TBP. }\end{array}\end{array}$

Patient's Needs of patients in TBP.

\section{Suggestions for future implementation strategies}

- Evaluate the perception of professionals regarding the recognition of the patient as the center of care and about the patient's needs are priorities in the institution.

- Develop diversified strategies for dissemination and training of the health team on the topic, such as virtual and printed materials, online and in loco training.

- Establish communities of practice to foster debate about the needs of patients in TBP.

- Identify if there is competitive pressure, that is, if the institution is influenced by the actions of another institution.

- Develop strategies to integrate experiences from other services.

III. INNER SETTING

\section{Implementation} Climate
The use of the same or similar intervention in other institutions such as benchmarking could positively influence the implementation
-When planning implementation, consider the political-economic status of the institution.

- Consider delaying implementation when identifying a climate incompatible with the intervention.

- Identify the degree of importance given to the intervention from an institutional perspective.

- Previously investigate the positive and facilitating impact that the intervention may bring to the problems perceived by individuals, especially health workers.

1 CFIR: Consolidated Framework for Implementation Research. 


\section{CFIR Domains Main lessons learned in the and Constructs implementation process}

Individual

Stage of

Change
Great variations in the degree of individual commitment to implementation hampered adherence to the Com-Efe protocol.

\section{Suggestions for future implementation strategies}

- Identify whether key stakeholders perceive the current situation as warranting change.

- Identify whether the individuals involved in the context are receptive to the idea of systematizing effective communication processes with patients in TBP.

- Identify the level of influence of each of the key stakeholders in the process in order to direct intervention planning.

\section{PROCESS}

Opinion

Leaders
The low level of leadership involvement negatively affected adherence by the other team members.
- Identify whether the intervention fits the organization's culture in terms of demands for leadership.

- Identify whether the use of the intervention is supported by leaders.

- Establish preliminary contacts with service leaders and include them in the implementation planning process.

1 CFIR: Consolidated Framework for Implementation Research.

Source: own authorship

\section{Discussion}

The implementation of Com-Efe was characterized as a failure since it was not incorporated into the routines of the medical clinic and surgical clinics during the study period. There are few publications dedicated to detailing the reasons for the failure in implementation, and this is one of the strengths of the present study. The science of implementation allows for an organized and in-depth documentation of barriers and facilitators identified throughout an implementation process to be done and can collaborate in the implementation of several innovations in similar contexts, as long as adaptations are made. However, failures in implementation processes are equally relevant from the perspective of institutional and collective learning ${ }^{19-21}$.

In our study, we attribute the failure of implementation to four main elements: the origin of the intervention, institutional incorporation, understanding of the concept of effective communication, and the context of the institution at the time of the study. These elements are not completely independent; on the contrary, they exerted complex influences on each other. 
Regarding the origin of the intervention, if on the one hand there was recognition and appreciation by the participants regarding the academic origin of the protocol, on the other hand the need for the intervention did not stem from institutional needs, affecting the feeling of belonging and the urgency of change. The results obtained suggest that, although the scientific evidence is recognized, the education of patients in TBP is not seen as a problem that deserves to be prioritized by the institution, which may be due to lack of time, shortage of staff, lack of standardization, malpractice or ignorance of the importance of such action ${ }^{22}$. Even when it is recognized as a priority, it does not seem to have any further reflection on the current process regarding its prescriptive or dialogic nature. Therefore, it is necessary to develop strategies that lead to this reflection, in addition to identifying the institution's level of expectation in reviewing the relationship between $\mathrm{HCW}$ and patients.

Despite being identified in relation to individual influence on implementation, there was a failure in the engagement of stakeholders for a new way of thinking about education for TBP, carried out with the aim of engaging patients in their own care ${ }^{23}$. Research has shown that HAI prevention behavior can be affected by the psychological status of individuals ${ }^{24}$. Therefore, assessing and identifying the main stakeholders on an individual basis can be the key to raising awareness needed to change attitudes and behaviors.

Throughout the implementation process, there was a gap in understanding the concept of effective communication. Most HCW remain focused on the use of traditional and prescriptive education models, which can be provided to patients and families without a real commitment to a dialogic attitude. Therefore, effective communication does not seem to have been incorporated and the dialogic component was not captured during the training stages.Patient-centered care remains more focused on using hard or soft-hard technologies and immediate problem-solving, while the lightweight technologies that produce effective communication have not been prioritised. It is necessary to make changes in the work process, through the effective use of light technologies and their articulation with other technologies.

Nevertheless, the idea of systematizing the educational process of patients in TBP seems to have been fully captured and perceived as a possible advantage in the qualification of nursing actions.

Effective communication among HCW, and between HCW and patients is essential for the implementation of routines and future interventions. Com-Efe can collaborate with effective communication between HCW and patients, as it is a systematized process, but at the same time allows for dialogue between the parties, in addition to considering individual elements of patients, aiming to prevent adverse events related to the TBP situation.

The full institutional incorporation of Com-Efe did not take place during the Com-Efe implementation process, for two main reasons. The first is related to the current context of the institution, in which the COVID-19 pandemic could represent a favorable moment for the full incorporation of Com-Efe, which, however, did not happen. We believe that, in addition to the institution's internal factors, the dramatic 
context of the Brazilian response to COVID-19 has had a negative influence ${ }^{25,26}$. The second is related to the fact that the leaders have not been strongly engaged with the implementation process. This was perceived by the frontline nurses, also reflecting in them an attitude of not prioritizing the subject. Leadership is recognized as an important indicator for the development of organizational culture and effective performance in the provision of healthcare. There is a strong relationship between leadership and safety, effectiveness and equity in care ${ }^{27}$.

In our study, we used the CFIR as a frame of reference for the methodological development and analysis of results. However, not all constructs proposed by Damschroder et al (2009) ${ }^{14}$ could be identified and addressed.

\section{Study limitations}

The concurrence of the pandemic with the development of the study was an uncontrolled element and certainly brought interference both in the implementation process and its assessment. Additionally, due to the high turnover of nurses, many of participants engaged in the implementation process from the beginning were no longer in the study settings, reducing the number of potential interviewees. However, we consider that these limitations are unavoidable in real-life studies and are part of the natural challenges of implementation processes.

\section{Conclusions}

The context in which the implementation was carried out proved to be complex, presenting barriers from the beginning of the process, which could not be overcome by the extant facilitating factors and by the implementation strategies adopted. In this study, we identified that one of the main barriers to the full implementation of Com-Efe was the difficulty in incorporating the central element, the concept of vulnerability, which seeks, through a dialogical relationship between HCW and patients, to reduce adverse events related to TBP. Relevant barriers referring to the institutional context also had a negative influence.

The lessons learned in this study allowed us to propose suggestions for future implementations in similar contexts. Among them are to develop strategies to generate awareness of patient-centered care, maintaining the patient's autonomy and seeking a dialogical process for the patient's engagement.

\section{List Of Abbreviations}




\begin{tabular}{|ll|}
\hline Com-Efe & Effective Communication \\
\hline SP & Standard Precautions \\
\hline TBP & Transmission-Based Precautions \\
MO & Microorganism \\
HAI & Healthcare associated infection \\
\hline HCW & HealthcareWorkers \\
\hline CFIR & Consolidated Framework for Implementation Research \\
\hline COVID-19 & Coronavirus Disease 2019 \\
\hline
\end{tabular}

\section{Declarations}

\section{Ethics approval and consent to participate}

The research project was approved by the Research Ethics Committee of the University of São Paulo School of Nursing and the University Hospital of University of São Paulo, and the Free and Informed Consent Terms were drawn up for the participants in accordance with resolution 466/12. CAEE: 80384517.5.0000.5392.

\section{Consent for publication}

Not applicable.

\section{Availability of data and materials}

Data available on request from the authors.

\section{Competing interests}

The authors declare no relevant conflict of interest related to the subject of this paper.

\section{Funding}

This study was partially financed by the"Coordenação de Aperfeiçoamento de Pessoal de Nível Superior - Brazil (CAPES)". Finance code: 001.

\section{Authors' contribution}

LFJ: carried out the research as her doctoral project, which was the basis for this article. She worked on research planning, data collection, data analysis, as well as writing the article.

RL: contribution to the writing and critical review of the article. 
AMF: contribution to writing and critical review of the article.

ST: contribution to the writing and critical review of the article.

MCP: general advisor of the research, contributed to the planning, and guided the collection and interpretation of data, plus discussion and critical review of the article.

\section{Acknowledgements}

We would like to acknowledge the infection prevention and control teams, and the nurses from education section, for their partnership in this study.

\section{References}

1. Siegel J, Rhinehart E, Jackson M, Chiarello L. 2007 Guideline for Isolation Precautions: Preventing Transmission of Infectious Agents in Healthcare Settings. Am J Infect Control 2007;35(10 Suppl 2):S65-S164.

2. Kramer S, Omonaiye O, Digby R, Berry D, Considine J, Dunning T, et al. An evaluation of interventions to improve outcomes for hospitalized patients in isolation: A systematic review. Am J Infect Control 2021.

3. Nair R, Perencevich EN, Goto M, Livorsi DJ, Balkenende E, Kiscaden E, et al. Patient care experience with utilization of isolation precautions: systematic literature review and meta-analysis. Clin Microbiol Infect. 2020;26(6):684-95.

4. Zahar JR, Garrouste-Orgeas M, Vesin A, Schwebel C, Bonadona A, Philippart F, et al. Impact of contact isolation for multidrug-resistant organisms on the occurrence of medical errors and adverse events. Intensive Care Med. 2013;39(12):2153-60.

5. Tran K, Bell C, Stall N, Tomlinson G, McGeer A, Morris A, et al. The Effect of Hospital Isolation Precautions on Patient Outcomes and Cost of Care: A Multi-Site, Retrospective, Propensity ScoreMatched Cohort Study. J Gen Intern Med. 2017;32(3):262-8.

6. Tartari E, Weterings V, Gastmeier P, Rodríguez Baño J, Widmer A, Kluytmans J, et al. Patient engagement with surgical site infection prevention: an expert panel perspective. Antimicrob Resist Infect Control. 2017;6:45.

7. Juskevicius LF, Padoveze MC. Specific precautions for avoiding microorganism transmission: development and validation of an educational guide. Cogit Enferm (Online). 2016;21(4):01-10.

8. Sharp D, Palmore T, Grady C. The ethics of empowering patients as partners in healthcare-associated infection prevention. Infect Control Hosp Epidemiol. 2014;35(3):307-9.

9. Pomey MP, Ghadiri DP, Karazivan P, Fernandez N, Clavel N. Patients as partners: a qualitative study of patients' engagement in their health care. PLoS One. 2015;10(4):e0122499.

10. Nilsen $P$, Bernhardsson S. Context matters in implementation science: a scoping review of determinant frameworks that describe contextual determinants for implementation outcomes. BMC 
Health Serv Res. 2019;19(1):189.

11. Butenko S, Lockwood C, McArthur A. Patient experiences of partnering with healthcare professionals for hand hygiene compliance: a systematic review. JBI Database System Rev Implement Rep. 2017;15(6):1645-70.

12. Hostiuc S, Molnar AJ, Moldoveanu A, Aluaş M, Moldoveanu F, Bocicor I, et al. Patient autonomy and disclosure of material information about hospital-acquired infections. Infect Drug Resist. 2018;11:369-75.

13. Owens K, Eggers J, Keller S, McDonald A. The imperative of culture: a quantitative analysis of the impact of culture on workforce engagement, patient experience, physician engagement, value-based purchasing, and turnover. J Healthc Leadersh. 2017;9:25-31.

14. Damschroder LJ, Aron DC, Keith RE, Kirsh SR, Alexander JA, Lowery JC. Fostering implementation of health services research findings into practice: a consolidated framework for advancing implementation science. Implement Sci. 2009;4:50.

15. World Health Organization. Global action plan on antimicrobial resistance. Geneva: World Health Organization; 2015. 28p. Available at: https://ahpsr.who.int/publications/i/item/global-action-planon-antimicrobial-resistance. In.

16. Breimaier HE, Heckemann B, Halfens RJ, Lohrmann C. The Consolidated Framework for Implementation Research (CFIR): a useful theoretical framework for guiding and evaluating a guideline implementation process in a hospital-based nursing practice. BMC Nurs. 2015;14:43.

17. Bardin L. Análise de conteúdo. São Paulo: Edições 70; 2016. 279p.

18. Tong A, Sainsbury P, Craig J. Consolidated criteria for reporting qualitative research (COREQ): a 32item checklist for interviews and focus groups. Int J Qual Health Care. 2007;19(6):349-57.

19. Kigume R, Maluka S. The failure of community-based health insurance schemes in Tanzania: opening the black box of the implementation process. BMC Health Serv Res. 2021;21(1):646.

20. Mann C, Shaw ARG, Guthrie B, Wye L, Man MS, Chaplin K, et al. Can implementation failure or intervention failure explain the result of the 3D multimorbidity trial in general practice: mixedmethods process evaluation. BMJ Open. 2019;9(11):e031438.

21. Katz J, Wandersman A, Goodman RM, Griffin S, Wilson DK, Schillaci M. Updating the FORECAST formative evaluation approach and some implications for ameliorating theory failure, implementation failure, and evaluation failure. Eval Program Plann. 2013;39:42-50.

22. Olino L, Gonçalves AC, Strada JKR, Vieira LB, Machado MLP, Molina KL, et al. Effective communication for patient safety: transfer note and Modified Early Warning Score. Rev Gaúcha Enferm. 2019;40(esp):e20180341.

23. Goodman MS, Sanders Thompson VL. The science of stakeholder engagement in research: classification, implementation, and evaluation. Transl Behav Med. 2017;7(3):486-91.

24. Assis GR, Benuti GG, Moretto MLT, Vidigal CG, Santos RPD, Lobo RD, et al. Psychological characteristics are associated with healthcare worker adherence to infection control practices. Am J Infect Control. 2021;49(7):879-84. 
25. Carneiro M, Hessel Dias VMC, Miranda Costa MM, Pinheiro D, Vidal CFL, Rodrigues O, et al. Coronavirus disease 2019 (COVID-19) Brazil Task Force: How to navigate troubled waters. Infect Control Hosp Epidemiol 2021 Feb 2:1-2. doi: 10.1017/ice.2021.41. Epub ahead of print. PMID: 33526163; PMCID: PMC8593370.:1-2.

26. Boschiero MN, Palamim CVC, Ortega MM, Mauch RM, Marson FAL. One Year of Coronavirus Disease 2019 (COVID-19) in Brazil: A Political and Social Overview. Annals of Global Health. 2021;87(1):44.

27. Santos JLG, Pin SB, Guanilo MEE, Balsanelli AP, Erdmann AL, Ross R. Nursing leadership and quality of care in a hospital setting: mixed methods research. Rev Rene. 2018;19:e3289.

\section{Figures}

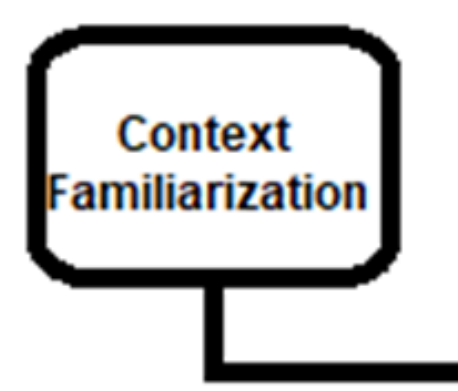

Phase 1

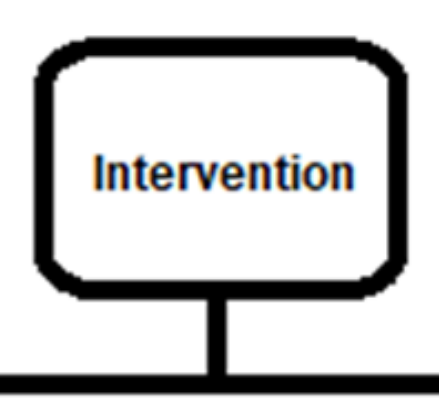

Phase 2

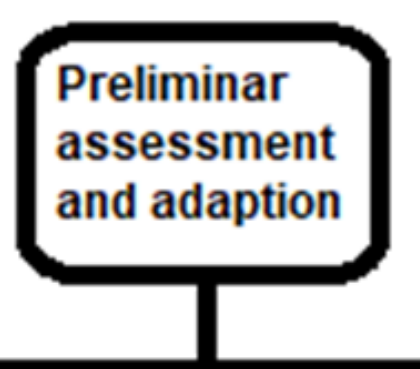

Phase 3
Final assessment assessment

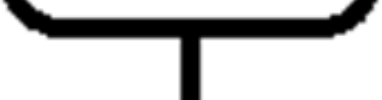

Phase 4

Figure 1

Schematic representation of the phases of the implementation process, provided by authors.

\section{Supplementary Files}

This is a list of supplementary files associated with this preprint. Click to download.

- COREQ.docx 